\title{
Risk Factors for the Development of High-risk Stigmata in Branch-duct Intraductal Papillary Mucinous Neoplasms
}

\author{
Tatsuhiro Yamazaki, Takeshi Tomoda, Hironari Kato, Kazuya Miyamoto, Akihiro Matsumi, \\ Eijiro Ueta, Yuki Fujii, Yosuke Saragai, Daisuke Uchida, Kazuyuki Matsumoto, \\ Shigeru Horiguchi, Koichiro Tsutsumi and Hiroyuki Okada
}

\begin{abstract}
:
Objective Strict follow-up is recommended for branch-duct intraductal papillary mucinous neoplasms (BDIPMNs) to avoid missing the development of high-risk stigmata (HRS) at a premalignant stage. This study explored the risk factors associated with the development of HRS during follow-up.

Methods We performed a retrospective analysis of 283 patients with BD-IPMN, treated at Okayama University Hospital in Japan between January 2009 and December 2016. Only patients with imaging studies indicative of classical features of BD-IPMN without HRS and followed for over one year were included in the study. We performed radiological follow-up every six months and collected patients' demographic data, cyst characteristics, and clinical outcomes and used univariate logistic regression models to determine the odds of developing HRS.

Results Ten patients (3.5\%) developed HRS after a median surveillance period of 55.8 months. The main pancreatic duct (MPD) size $(5-9 \mathrm{~mm})$ and cyst growth rate $(>2.5 \mathrm{~mm} /$ year) were both suggested to be possible risk factors for the development of HRS [odds ratio, 14.2; 95\% confidence interval (CI), 3.1-65.2, $\mathrm{p}=$ 0.0006 , and odds ratio, 6.1; 95\% CI 1.5-25.5, $\mathrm{p}=0.014]$. Regarding the number of worrisome features (WFs), the rate of HRS development was $2.0 \%(4 / 199)$ in cases with no WF, $1.6 \%(1 / 62)$ in cases with single WF and $22.7 \%(5 / 22)$ in cases with multiple WFs, respectively. The rate of HRS development was significantly higher in cases with multiple WFs than in the other cases $(\mathrm{p}<0.0001)$.

Conclusion MPD dilation, rapid cyst growth, and multiple WFs were significant risk factors for the development of HRS. In the presence of such features, it is necessary to closely follow the development of HRS and avoid missing the best opportunity to perform surgical intervention.
\end{abstract}

Key words: surveillance, prognosis factor, intraductal papillary mucinous neoplasm, pancreatic cancer

(Intern Med 60: 3205-3211, 2021)

(DOI: 10.2169/internalmedicine.7168-21)

\section{Introduction}

The detection of intraductal papillary mucinous neoplasms (IPMNs) of the pancreas has increased due to the use of cross-sectional imaging, such as computed tomography (CT), magnetic resonance imaging (MRI), magnetic resonance cholangiopancreatography (MRCP), and endoscopic ultrasound (EUS) $(1,2)$. However, the type of case determines whether or not resection is required and how follow- up should be performed.

The 2017 International Association of Pancreatology guideline (Fukuoka guideline) classifies clinical and radiological findings predicting malignancy into "high-risk stigmata (HRS)" and "worrisome features (WFs)" (3). HRS include i) obstructive jaundice, ii) enhancing mural nodule $\geq 5$ $\mathrm{mm}$, and iii) main pancreatic duct (MPD) $\geq 10 \mathrm{~mm}$, with surgical resection recommended for patients with any findings due to the high prevalence of cancer. WFs include i) a history of pancreatitis, ii) cyst $\geq 3 \mathrm{~cm}$, iii) enhancing mural 
nodule $<5 \mathrm{~mm}$, iv) thickened/enhancing cyst walls, v) MPD size 5-9 mm, vi) abrupt change in caliber of the pancreatic duct with distal pancreatic atrophy, vii) lymphadenopathy, viii) increased serum level of CA19-9, and ix) a cyst growth rate $\geq 5 \mathrm{~mm} / 2$ years. Features viii and ix were recently added as new WFs in the IPMN International Guidelines for 2017 (Fukuoka Guideline). In cases of branch-duct IPMN (BDIPMN), it is also necessary to be aware of the tumor markers and the rate cyst growth.

Strict follow-up is recommended for cases without HRS, and it seems important to not miss the development of HRS at a premalignant stage (4). However, which factors are associated with the development of HRS during follow-up is unclear.

The present study explored which WFs of BD-IPMN were risk factors for the development of HRS during followup.

\section{Materials and Methods}

\section{Study design}

This single-center retrospective study examined the predictive factors for the development of HRS from BD-IPMN during follow-up. Informed consent was obtained from all participants. The study protocol was approved by the ethical guidelines of the World Medical Association Declaration of Helsinki and was approved by the Okayama University Ethics Committee.

\section{Patients and the diagnosis of BD-IPMN}

Patients were identified from our EUS database, and consecutive patients with a clinical diagnosis of BD-IPMN at the Okayama University Hospital with a minimum of 12 months follow-up between January 2009 and December 2016 were included in this study. Patients were those with at least one cross-sectional imaging study (CT, MRI/MRCP, and EUS) performed three months or longer after the initial diagnosis. If there were no contraindications, EUS, contrastenhanced CT (CE-CT), and MRCP were performed in all cases at the initial diagnosis. BD-IPMN was defined as the presence of unilocular or multicystic lesions $\geq 5 \mathrm{~mm}$ in size communicating with the MPD, as clearly demonstrated by CT, MRCP, or EUS. When EUS revealed a suspected nodule in the cystic lesions, contrast-enhanced EUS (CE-EUS) using an ultrasound contrast agent (Sonazoid ${ }^{\mathrm{TM}}$; DaiichiSankyo, Tokyo, Japan) was performed. If there was an absence of blood flow signals in the intracystic structures, we diagnosed it as a protein plaque.

All patients with HRS at the initial diagnosis or with cysts suggestive of another diagnosis besides BD-IPMN (e.g., serous cystadenoma, mucinous cystic neoplasm, cystic neuroendocrine tumor, solid pseudopapillary tumor, or pseudocyst) were excluded from the study.

\section{Follow-up and data collection}

Patients diagnosed with BD-IPMN underwent radiological follow-up by CE-CT or MRCP alternately every six months, and cyst characteristics, including the location, maximum cyst size, solid component with or without enhancement, thickened cyst walls, MPD size, lymphadenopathy, and abrupt change in MPD caliber with distal pancreatic atrophy, were examined. The CA19-9 serum levels were routinely measured. The diameter of the cyst and MPD size were defined as the maximum diameter measured by MRCP, as recommended by the Fukuoka guidelines for evaluating pancreatic cysts $(3,5,6)$. In multifocal cases, the largest cyst was used. Thickened cyst walls were defined in cases with cyst walls thicker than $2 \mathrm{~mm}$. Increased serum levels of CA19-9 were defined in cases with levels $>40 \mathrm{U} / \mathrm{mL}$. If findings such as an increased cyst diameter or appearance of nodules were suspected on CE-CT or MRCP, EUS was performed. The follow-up period was set as the time between the initial cyst diagnosis (the first cross-sectional imaging on which the cyst was detected) and one of the following endpoints: the date of the last visit or death for patients without development of HRS; date of HRS development; or the date of the first discovery of malignancy on imaging for patients with an unresectable tumor.

We recommended surgical resection for cases in which HRS developed during follow-up. For other patients with a solid mass suspected of being pancreatic cancer or rapid growth in the cyst size, we considered surgical resection. Some patients who met the criteria for resection did not undergo surgery due to severe comorbidities or patient preference.

\section{Assessments}

The primary outcome was to evaluate the risk factors for the development of HRS in patients with BD-IPMN without HRS at the initial diagnosis. The evaluated risk factors were as follows: a history of pancreatitis, cyst $\geq 3 \mathrm{~cm}$, enhancing mural nodule $<5 \mathrm{~mm}$, thickened/enhancing cyst walls, MPD size 5-9 mm, abrupt change in caliber of the pancreatic duct with distal pancreatic atrophy, lymphadenopathy, increased serum level of CA19-9 ( $>40 \mathrm{U} / \mathrm{mL}$ ), and cyst growth rate $\geq 5$ $\mathrm{mm} / 2$ years, which is defined as a WF by the Fukuoka guidelines. The cyst growth rate was calculated as the cyst size at last follow-up - cyst size at the initial diagnosis/ follow-up period; an increase of $\geq 2.5 \mathrm{~mm} /$ year was defined as a WF. Furthermore, the initial cyst size and cyst growth rate were compared, and cases were assigned to four different groups according to the cyst size.

\section{Statistical analyses}

Continuous variables were presented as the mean with standard deviation (SD) or the median with range when appropriate, and categorical variables were expressed as frequencies with percentages. Wilcoxon's rank sum test was performed to compare the continuous variables, and Fisher's 


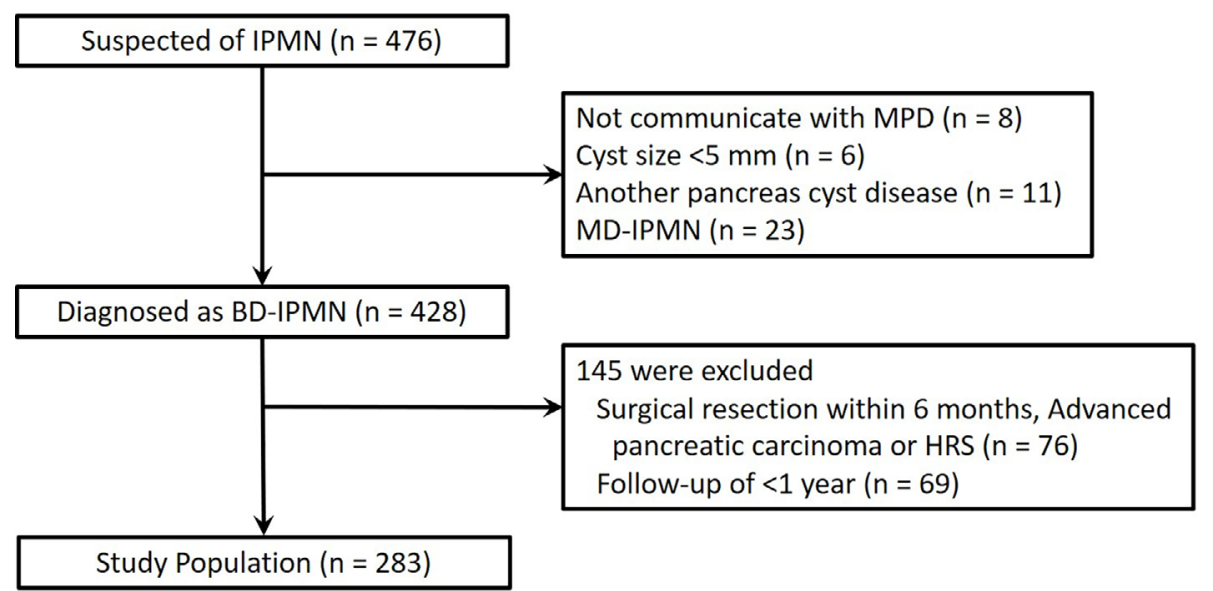

Figure 1. Flow diagram of the study population.

exact test was performed to evaluate the categorical variables. A p value $<0.05$ was considered statistically significant. Univariate analyses were performed to predict the odds ratio (OR) of developing HRS. A multivariate model was not feasible because of the small sample size of the HRS group. All statistical analyses were performed using the $\mathrm{JMP}^{\circledR}$ software program, version 14 (SAS Institute, Cary, USA).

\section{Results}

\section{Patient characteristics}

A total of 476 patients who underwent EUS for suspicious IPMN on CT/MRCP were identified during the study period. Among these patients, 48 were excluded for the following reasons: no communication with MPD $(n=8)$, cyst size $<5 \mathrm{~mm}(\mathrm{n}=6)$, possibility of another pancreatic cyst disease $(n=11)$, and main duct IPMN $(n=23)$. A total of 428 patients were diagnosed with BD-IPMN. However, those who underwent surgical resection within 6 months or who had been diagnosed with advanced pancreatic ductal adenocarcinoma (PDAC) or HRS at the initial diagnosis $(n=76)$ and with follow-up periods of $<1$ year $(n=69)$ were excluded from the study. Ultimately, 283 patients were included in this analysis (Fig. 1).

The baseline characteristics are summarized in Table 1. In this study, 139 men and 144 women with a mean age of 66.3 years were included. The median follow-up duration was 53.3 months (13.7-121.6). The WF excluding cyst growth rate $>2.5 \mathrm{~mm}$, which could not be identified at the initial diagnosis, was detected in 89 WFs of 76 patients at the initial diagnosis.

\section{Morphological data at the initial diagnosis and dur- ing follow-up}

The mean initial cyst size was $18.0 \mathrm{~mm}$, and the MPD was $2.5 \mathrm{~mm}$. At the final follow-up examination, the mean cyst size was $20.4 \mathrm{~mm}$, and the MPD was $2.8 \mathrm{~mm}$. The mean annual rate of cyst growth was $0.57 \mathrm{~mm}$, and the size in 21 cases $(7.4 \%)$ increased by more than $2.5 \mathrm{~mm} / \mathrm{year}$, which is a WF (Table 2). Among these cases, eight had no WFs at the initial diagnosis. A total of 110 WFs in 84 patients were ultimately detected at the initial diagnosis, including a cyst growth rate $>2.5 \mathrm{~mm} /$ year.

\section{Development of HRS and risk factors}

Of the 283 patients, 10 (3.5\%) developed HRS after a median surveillance period of 55.8 months (range, 30.4112.3) before HRS development, including an enhancing mural nodule $\geq 5 \mathrm{~mm}(\mathrm{n}=7)$, MPD size $\geq 10 \mathrm{~mm} \quad(\mathrm{n}=2)$, and enhancing solid component $\geq 5 \mathrm{~mm}$ with an MPD size $\geq 10$ $\mathrm{mm}(\mathrm{n}=1)$. No patients developed obstructive jaundice. Among these patients, five patients developed HRS after a five-year surveillance period.

We evaluated which WF was a potential risk factor for the development of HRS in this study (Table 3). The MPD size $(5-9 \mathrm{~mm})$ and cyst growth rate $(>2.5 \mathrm{~mm} / \mathrm{year})$ were both suggested as possible risk factors for the development of HRS (OR 14.2, 95\% CI 3.1-65.2, p=0.0006 and OR 6.1, 95\% CI 1.5-25.5, $\mathrm{p}=0.014)$. An increased serum level of CA 19-9 (>40 U/mL) at the diagnosis was not a feature of HRS development $(\mathrm{p}=0.20)$.

Subsequently, we examined whether or not the simultaneous presence of WF at the initial diagnosis could be used as a predictive marker of HRS development during follow-up. For each patient, the number of WFs was counted, and the rate of HRS development was compared (Fig. 2). When the patients were classified into groups with no WFs, a single WF, and multiple WFs, HRS development was seen in 4 of $199(2.0 \%)$ patients with no WFs, 1 of $62(1.6 \%)$ patients with a single WF, and 5 of $22(22.7 \%)$ patients with multiple WFs. The multiple WF group showed a significantly higher incidence rate of HRS than the other groups, and its OR was 15.1 [95\% confidence interval (CI): 4.0-57.1; p= $0.0003]$.

\section{Morphological changes in cysts in terms of initial size}

The participants were categorized into 4 groups according 
Table 1. Clinical Characteristics of Patients with Branch-duct Intraductal Mucinous Papillary Neoplasms.

\begin{tabular}{lc}
\hline \multicolumn{1}{c}{$\mathrm{n}=283$} & $\mathrm{n}(\%)$ \\
\hline Gender & $139(49.1)$ \\
Male & $144(50.9)$ \\
Female & $66.3 \pm 10.5$ \\
Age (mean \pm SD), year & \\
Cyst location & $140(49.5)$ \\
Head & $86(30.4)$ \\
Body & $57(20.0)$ \\
Tail & \\
Cyst morphology & $36(12.7)$ \\
Unilocular & $247(87.3)$ \\
Multicystic & $162(57.2)$ \\
Multifocal disease & $18.0 \pm 9.9$ \\
Initial cyst size (mean \pm SD), mm & $2.5 \pm 1.1$ \\
MPD (mean \pm SD), mm & $20.3 \pm 44.9$ \\
CA19-9 (mean \pm SD), U/mL & $53.3(13.7-121.6)$ \\
Follow-up periods, median (range), month & \\
Patients with WFs & $76(26.9)$ \\
Counts of WFs at the initial diagnosis & 89 \\
Pancreatitis & 5 \\
Thickened cyst walls & 4 \\
Cyst size $>3$ cm & 38 \\
Mural nodule $<5$ mm & 4 \\
MPD 5-9 mm & 11 \\
Abrupt change in caliber of PD with distal atrophy & 1 \\
Lymphoadenopathy & 2 \\
CA19-9 $>40$ U/mL & 24 \\
\hline
\end{tabular}

SD: standard deviation, MPD: main pancreatic duct, CA19-9: carbohydrate antigen 19-9, WF: worrisome feature, PD: pancreatic duct

Table 2. Morphological Changes in Cysts $(n=283)$.

\begin{tabular}{lcc}
\hline & Initial & Final \\
\hline Cyst size (mean $\pm \mathrm{SD}), \mathrm{mm}$ & $18.0 \pm 9.9$ & $20.4 \pm 12.4$ \\
Cyst growth rate $(\mathrm{mean} \pm \mathrm{SD}), \mathrm{mm} / \mathrm{y}$ & & $0.57 \pm 1.14$ \\
Cyst growth rate $\geq 2.5 \mathrm{~mm} / \mathrm{y}, \mathrm{n}(\%)$ & & $21(7.4)$ \\
MPD (mean $\pm \mathrm{SD}), \mathrm{mm}$ & $2.5 \pm 1.1$ & $2.8 \pm 1.6$ \\
CA19-9 (mean $\pm \mathrm{SD}), \mathrm{U} / \mathrm{mL}$ & $20.3 \pm 44.9$ & $20.3 \pm 41.1$ \\
\hline
\end{tabular}

SD: standard deviation, MPD: main pancreatic duct, CA19-9: carbohydrate antigen 19-9

to their initial cyst size: group 1, $<10 \mathrm{~mm}(\mathrm{n}=51)$; group 2,

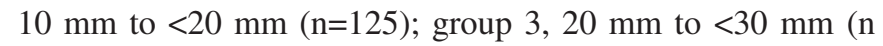
=69); and group 4, $\geq 30 \mathrm{~mm}(\mathrm{n}=38)$. The respective median annual growth rates for groups $1,2,3$, and 4 were $0 \mathrm{~mm}$, $0.20 \mathrm{~mm}, 0.40 \mathrm{~mm}$, and $0.98 \mathrm{~mm}$ (Fig. 3). The annual growth rates were significantly different among the 4 groups $(\mathrm{p}<0.001)$. However, the incidence of newly developed HRS was not related to the initial cyst size [0\% (0/51), 2.4\% (3/ $125), 5.8 \%(4 / 69)$, and $7.9 \%(3 / 38)$ in groups $1,2,3$, and 4 , respectively; $\mathrm{p}=0.11$ ].

\section{Surgery and progress}

Of the 10 patients who developed HRS, 3 underwent sur- gery (diagnosed with IPMN-derived invasive carcinoma in 2 and IPMN with low-grade dysplasia in 1), and 1 was found to have pancreatic cancer with bone metastases. The other six patients continued to receive follow-up because of severe comorbidities or the patient's preference. In addition, regarding the patients who developed HRS, 5 (1.8\%) underwent surgery, including 2 for suspected concomitant PDAC and 3 due to the patient's wish in response to cyst size enlargement after a mean surveillance period of 62.4 months. The two with suspected concomitant PDAC were pathologically diagnosed PDAC, while the other three were diagnosed with IPMN with low-grade dysplasia.

\section{Discussion}

Previous reports have shown that the presence of HRS is associated with a $40 \%$ risk of IPMN-related death, reinforcing the notion that surgical resection should be offered to fit patients (7). In cases of IPMN with HRS, the 3-year pancreatic carcinoma risks of obstructive jaundice, an enhancing solid component, and MPD >10 mm were 79.8\%, 37.3\%, and $39.4 \%$, respectively (4). Thus, in the follow-up of BDIPMN, HRS is considered the most important factor for predicting the appearance of IPMN-derived carcinoma. In the 
Table 3. Risk Factors for the Development of HRS.

\begin{tabular}{lcccc}
\hline \multicolumn{1}{c}{$\mathrm{n}(\%)$} & $\begin{array}{c}\text { Development of HRS } \\
\mathrm{n}=10\end{array}$ & $\begin{array}{c}\text { Not HRS } \\
\mathrm{n}=273\end{array}$ & Odds ratio (95\% CI) & p value \\
\hline Pancreatitis & $0(0)$ & $5(1.8)$ & 0 & 0.99 \\
Thickened/enhanced cyst walls & $1(10.0)$ & $3(1.1)$ & $10.0(0.9-105.8)$ & 0.06 \\
Cyst size $>3 \mathrm{~cm}$ & $3(30.0)$ & $35(12.8)$ & $2.9(0.7-11.8)$ & 0.14 \\
Mural nodule $<5 \mathrm{~mm}$ & $1(10.0)$ & $3(1.1)$ & $10.0(0.9-105.8)$ & 0.06 \\
MPD 5-9 mm & $3(30.0)$ & $8(2.9)$ & $14.2(3.1-65.2)$ & 0.0006 \\
Abrupt change in caliber of PD with distal atrophy & $0(0)$ & $1(0.4)$ & 0 & 0.99 \\
Lymphadenopathy & $0(0)$ & $2(0.7)$ & 0 & 0.99 \\
CA19-9 $>$ 40 U/mL & $2(20.0)$ & $22(8.1)$ & $2.9(0.6-14.3)$ & 0.20 \\
Cyst growth rate $>2.5 \mathrm{~mm} / \mathrm{y}$ & $3(30.0)$ & $18(6.6)$ & $6.1(1.5-25.5)$ & 0.014 \\
\hline
\end{tabular}

HRS: high-risk stigmata, CI: confidence interval, MPD: main pancreatic duct, PD: pancreatic duct, CA19-9: carbohydrate antigen 19-9

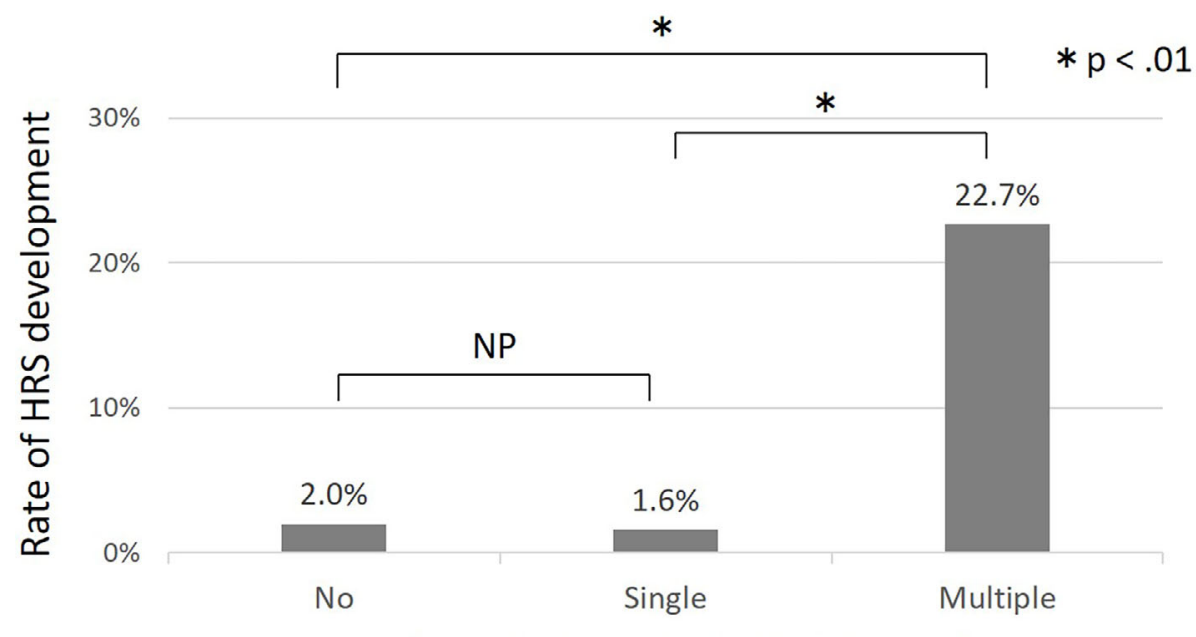

Number of WF at the initial diagnosis

Figure 2. Relationship between the number of WFs at the initial diagnosis and the rate of HRS development. WF: worrisome features, HRS: high-risk stigmata

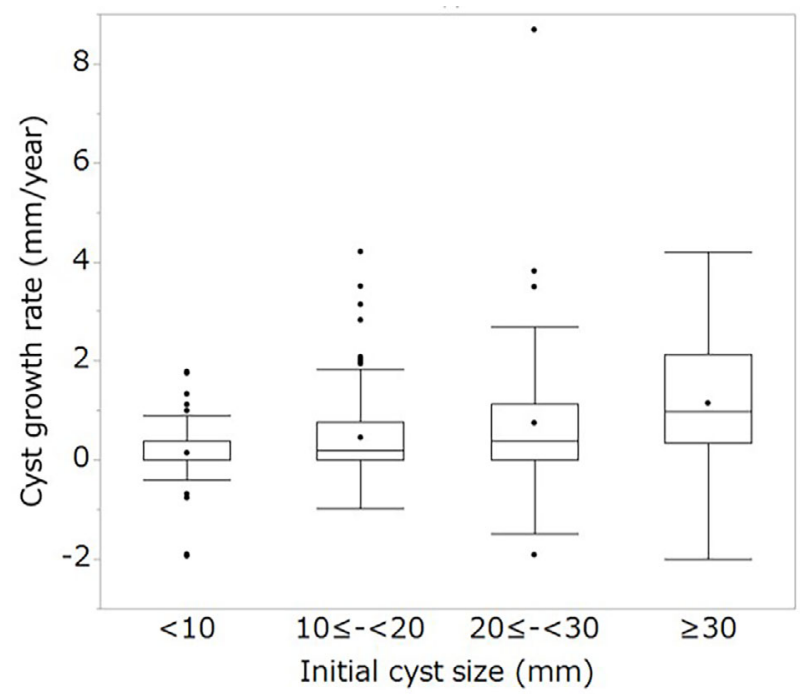

Figure 3. Depiction of cyst growth rate according to initial cyst size. The median annual cyst growth rate was $0.57 \mathrm{~mm}$. The larger the cyst size at the diagnosis, the faster the cyst growth rate increased. present study, we explored the risk factors associated with the development of HRS during follow-up.

Reports have evaluated the risk factors of malignancy associated with individual cyst features of BD-IPMN, and a cyst size $>3 \mathrm{~cm}$, presence of a mural nodule, and MPD dilatation $>5 \mathrm{~mm}$ proved to be the strongest predictors of pancreatic malignancy $(8,9)$.

In our study, in addition to dilation of the MPD, rapid cyst growth and multiple WFs were found to be factors potentially associated with the development of HRS. Kang et al. reported that cysts growing faster than $2 \mathrm{~mm} / \mathrm{year}$ presented a significantly higher risk of malignancy in all 201 subjects with an initial cyst size of $<30 \mathrm{~mm}$ without MPD dilation and no mural nodule than more slowly growing cysts (10). Kolb et al. studied a group of 188 patients with low-risk IPMN, and 12 developed WF. Among them, the rate of BD-IPMN growth was greater in patients who developed WF than in those who did not $(2.84 \mathrm{~mm} / \mathrm{year}$ vs. 0.23 $\mathrm{mm} /$ year; $\mathrm{p}<0.001)(11)$. In a recent study, a rapid cyst growth rate $(\geq 2.5 \mathrm{~mm} /$ year) was the main predictor of malignancy development in presumed BD-IPMN without WFs or HRS (12). In the present study, of the 21 patients with a 
cyst growth rate $\geq 2.5 \mathrm{~mm} /$ year, $3(14.3 \%)$ developed HRS. Although most BD-IPMNs are indolent and dormant, some cysts rapidly grow with the development of HRS. During surveillance of BD-IPMN, particular focus should be placed on the cyst growth rate.

In a recently published multi-institutional study by Wilson et al., high-grade dysplasia and invasive carcinoma were found in $57.4 \%$ of patients with multiple WFs, $31.1 \%$ with a single WF, and $24.6 \%$ with no WFs, among surgical cases of IPMN. This study indicated that the number of WFs was associated with malignancy of BD-IPMN (13). Other studies have scored the morphology of IPMN and created risk models for malignancy, arguing that the larger the nodule diameter, cyst size, and MPD diameter, the higher the risk of malignancy (14). Consistent with these studies, the group with multiple WFs in the present study developed HRS more frequently than other groups. However, another study found that the WF counts were not related to the risk of malignant IPMN. In surgical cases, the mean WF counts were 1.52, $2.11,2.14$, and 2.20 for low-, intermediate-, and high-grade dysplasia and invasive carcinoma, respectively (15). Therefore, additional confirmatory studies are necessary to validate our results.

Cyst size $>3 \mathrm{~cm}$ was not a significant factor in the development of HRS, but larger cysts had a greater cyst growth rate than smaller ones in this study. Han et al. reported that larger cysts, particularly those larger than $2 \mathrm{~cm}$, showed significantly faster annual growth rates, and patients with initially larger cysts developed more WFs during surveillance than others (16). Thus, the initial cyst size is an important factor for the follow-up of BD-IPMN as it is related to the rate of cyst growth. Although a mural nodule, which has been reported to be associated with cancer in many reports, was not extracted as a risk factor, our study had too little power to detect differences due to the small number of cases with a mural nodule at the initial diagnosis $(16,17)$.

The American Gastroenterological Association guidelines recommend discontinuing surveillance of non-progressive IPMNs within five years (18). Conversely, accumulating evidence suggests that patients with BD-IPMN may remain at high risk of developing pancreatic carcinoma even after fiveyear surveillance (19-21). In a Japanese study involving 804 patients with BD-IPMNs followed for $>5$ years, the overall cumulative incidence of pancreatic cancer was $3.5 \%$ at 10 years from the initial diagnosis (21). In a study at a US referral center, high-grade dysplasia and invasive carcinoma were observed in $20(5.5 \%)$ and $16(4.4 \%)$ patients, respectively, among 363 patients included after 5 years of followup (22). Consistent with these studies, 5 out of the 10 patients who developed HRS in our study showed HRS after 5 years of follow-up, supporting the prolonged surveillance of patients with BD-IPMN. Further investigations are warranted to create surveillance programs based on these results.

Several limitations associated with the present study warrant mention. First, this was a retrospective single-center study with a small sample size. Therefore, the possibility of unintentional selection bias in the selection of patients could not be fully excluded. Second, there were only 10 patients among the 285 included who met the primary outcome (development of HRS). Due to the small outcome number, a multivariate analysis was not performed. A further study in a large patient cohort with a long follow-up period is required to validate our results.

In conclusion, this study shows that among BD-IPMN patients without HRS at the initial diagnosis, 3.5\% ultimately developed HRS during surveillance, and the dilation of the MPD, rapid cyst growth, and multiple WFs were suggested to be possible risk factors for the development of HRS. In cases with such features, it is necessary to closely follow the development of HRS and be careful not to miss the timing of surgery.

\section{The authors state that they have no Conflict of Interest (COI).}

\section{Acknowledgement}

We would like to thank Dr. Toshiharu Mitsuhashi for his statistical advice.

\section{References}

1. Klibansky DA, Reid-Lombardo KM, Gordon SR, Gardner TB. The clinical relevance of the increasing incidence of intraductal papillary mucinous neoplasm. Clin Gastroenterol Hepatol 10: 555558, 2012.

2. Bassi C, Sarr MG, Lillemoe KD, Reber HA. Natural history of intraductal papillary mucinous neoplasms (IPMN): current evidence and implications for management. J Gastrointest Surg 12: 645650, 2008.

3. Tanaka M, Fernandez-Del Castillo C, Kamisawa T, et al. Revisions of international consensus Fukuoka guidelines for the management of IPMN of the pancreas. Pancreatology 17: 738-753, 2017.

4. Mukewar S, de Pretis N, Aryal-Khanal A, et al. Fukuoka criteria accurately predict risk for adverse outcomes during follow-up of pancreatic cysts presumed to be intraductal papillary mucinous neoplasms. Gut 66: 1811-1817, 2017.

5. Zhang XM, Mitchell DG, Dohke M, Holland GA, Parker L. Pancreatic cysts: depiction on single-shot fast spin-echo MR images. Radiology 223: 547-553, 2002.

6. Berland LL, Silverman SG, Gore RM, et al. Managing incidental findings on abdominal CT: white paper of the ACR incidental findings committee. J Am Coll Radiol 7: 754-773, 2010.

7. Crippa S, Bassi C, Salvia R, et al. Low progression of intraductal papillary mucinous neoplasms with worrisome features and highrisk stigmata undergoing non-operative management: a mid-term follow-up analysis. Gut 66: 495-506, 2017.

8. Anand N, Sampath K, Wu BU. Cyst features and risk of malignancy in intraductal papillary mucinous neoplasms of the pancreas: a meta-analysis. Clin Gastroenterol Hepatol 11: 913-921, quiz e959-960, 2013.

9. Petrone MC, Magnoni P, Pergolini I, et al. Long-term follow-up of low-risk branch-duct IPMNs of the pancreas: is main pancreatic duct dilatation the most worrisome feature? Clin Transl Gastroenterol 9: 158, 2018.

10. Kang MJ, Jang JY, Kim SJ, et al. Cyst growth rate predicts malignancy in patients with branch duct intraductal papillary mucinous neoplasms. Clin Gastroenterol Hepatol 9: 87-93, 2011.

11. Kolb JM, Argiriadi $P$, Lee $K$, et al. Higher growth rate of branch duct intraductal papillary mucinous neoplasms associates with 
worrisome features. Clin Gastroenterol Hepatol 16: 1481-1487, 2018.

12. Marchegiani G, Andrianello S, Pollini T, et al. "Trivial" cysts redefine the risk of cancer in presumed branch-duct intraductal papillary mucinous neoplasms of the pancreas: a potential target for follow-up discontinuation? Am J Gastroenterol 114: 1678-1684, 2019.

13. Wilson GC, Maithel SK, Bentrem D, et al. Are the current guidelines for the surgical management of intraductal papillary mucinous neoplasms of the pancreas adequate? A multi-institutional study. J Am Coll Surg 224: 461-469, 2017.

14. Shimizu Y, Hijioka S, Hirono S, et al. New model for predicting malignancy in patients with intraductal papillary mucinous neoplasm. Ann Surg 2018.

15. Aso T, Ohtsuka T, Matsunaga T, et al. "High-risk stigmata" of the 2012 international consensus guidelines correlate with the malignant grade of branch duct intraductal papillary mucinous neoplasms of the pancreas. Pancreas 43: 1239-1243, 2014.

16. Han Y, Lee H, Kang JS, et al. Progression of pancreatic branch duct intraductal papillary mucinous neoplasm associates with cyst size. Gastroenterology 154: 576-584, 2018.

17. Seo N, Byun JH, Kim JH, et al. Validation of the 2012 International Consensus Guidelines using computed tomography and magnetic resonance imaging: branch duct and main duct intraductal papillary mucinous neoplasms of the pancreas. Ann Surg 263 $557-564,2016$
18. Vege SS, Ziring B, Jain R, Moayyedi P; Clinical Guidelines Committee. American Gastroenterological Association institute guideline on the diagnosis and management of asymptomatic neoplastic pancreatic cysts. Gastroenterology 148: 819-822; quize12-13, 2015.

19. Imbe K, Nagata N, Hisada $\mathrm{Y}$, et al. Validation of the American Gastroenterological Association guidelines on management of intraductal papillary mucinous neoplasms: more than 5 years of follow-up. Eur Radiol 28: 170-178, 2018.

20. Oyama H, Tada M, Takagi $K$, et al. Long-term risk of malignancy in branch-duct intraductal papillary mucinous neoplasms. Gastroenterology 158: 226-237.e5, 2020.

21. Khannoussi W, Vullierme MP, Rebours V, et al. The long term risk of malignancy in patients with branch duct intraductal papillary mucinous neoplasms of the pancreas. Pancreatology 12: 198-202, 2012.

22. Pergolini I, Sahora K, Ferrone CR, et al. Long-term risk of pancreatic malignancy in patients with branch duct intraductal papillary mucinous neoplasm in a referral center. Gastroenterology 153 : 1284-1294.e1, 2017.

The Internal Medicine is an Open Access journal distributed under the Creative Commons Attribution-NonCommercial-NoDerivatives 4.0 International License. To view the details of this license, please visit (https://creativecommons.org/licenses/ by-nc-nd/4.0/).

(C) 2021 The Japanese Society of Internal Medicine

Intern Med 60: 3205-3211, 2021 